\title{
Computation of free energy differences through nonequilibrium stochastic dynamics: the reaction coordinate case
}

\author{
Tony Lelièvre ${ }^{1}$, Mathias Rousset ${ }^{1}$, Gabriel Stoltz ${ }^{1,2}$ \\ 1: CERMICS, Ecole Nationale des Ponts et Chaussées (ParisTech), \\ 6 \& 8 Av. Pascal, 77455 Champs-sur-Marne, France. \\ 2: CEA/DAM Ile-de-France, BP 12, 91680 Bruyères-le-Châtel, France. \\ \{lelievre,stoltz\}@cermics.enpc.fr_mathias.rousset@polytechnique.org
}

July 11, 2021

\begin{abstract}
The computation of free energy differences through an exponential weighting of out-ofequilibrium paths (known as the Jarzynski equality [15, 16]) is often used for transitions between states described by an external parameter $\lambda$ in the Hamiltonian. We present here an extension to transitions between states defined by different values of some reaction coordinate, using a projected Brownian dynamics. In contrast with other approaches (see e.g. [22]), we use a projection rather than a constraining potential to let the constraints associated with the reaction coordinate evolve. We show how to use the Lagrange multipliers associated with these constraints to compute the work associated with a given trajectory. Appropriate discretizations are proposed. Some numerical results demonstrate the applicability of the method for the computation of free energy difference profiles.
\end{abstract}

Keywords: free energy, mean force, constrained dynamics, sampling techniques, Jarzynski equality, Feynman-Kac formula.

The free energy of a system is a quantity of paramount importance in statistical physics. It is of the form

$$
F=-\beta^{-1} \ln Z,
$$

where $\beta=1 /\left(k_{\mathrm{B}} T\right)$ ( $T$ denotes the temperature and $k_{\mathrm{B}}$ the Boltzmann constant) and $Z$ is the partition function

$$
Z=\int_{\Sigma} \exp (-\beta V) d \mu
$$

of the Boltzmann (or Gibbs) measure $\exp (-\beta V) d \mu$. In this expression, the function $V \equiv V(q)$ is the potential energy of the system (denoting by $q$ the position vector) and $\mu$ is a reference positive measure with support $\Sigma$. The space $\Sigma$ is the configuration space of the system. We will consider here that $\Sigma$ is a submanifold of $\mathbb{R}^{3 N}$, but all the results extend to the case when $\Sigma$ is a submanifold of $\mathbb{T}^{3 N}$ (the $3 N$-dimensional torus, which arises when using periodic boundary conditions). The statistics of the system are completely defined by $(V, \mu)$.

In most cases, $(V, \mu)$ is labeled using a $d$-dimensional parameter $z$ (with $d \ll 3 N$ ) which characterizes the system at some coarser level. The parameter $z$ can be independent of the 
current configuration of the system. In this case, only the expression of the potential $V$ depends on the parameter, so that the associated switching has sometimes been called 'alchemical transition'. Some examples of such parameters are the intensity of an external magnetic field for a spin system, or the temperature for a simulated annealing process. However, it is often the case that the parameter $z$ labels submanifolds of the configuration space, through level sets $\Sigma_{z}=\{\xi(q)=z\}$ of some function $\xi$. The function $\xi$ is called a 'reaction coordinate'. In this case, $\mu$ (especially the support of $\mu$ ) depends on $z$ and is defined using the orthogonal projection from $\mathbb{R}^{3 N}$ or $\mathbb{T}^{3 N}$ to $\Sigma_{z}$ (this will be made precise in Section 1.1). Standard examples of reaction coordinates are bond lengths or dihedral angles in a molecule.

The absolute free energy (11) can be computed only for certain systems, such as ideal gases, or solids at low temperature (resorting to the phonon spectrum) [23]. However, in many applications, the quantity of interest is the free energy difference between an initial and a final state (characterized by two different values of the parameter $z$ ). The free energy difference profiles indeed give information about the relative stabilities of several species, as well as their transition kinetics. The free energy differences are much more amenable to compute than the absolute free energy. Classical techniques to this end fall within three main classes. The first one, dating back to Kirkwood [17, is thermodynamic integration, which mimics the quasi-static evolution of a system as a succession of equilibrium samplings, which amounts to an infinitely slow switching between the initial and final states. The second one, the free energy perturbation method, was introduced by Zwanzig [35. It recasts free energy differences as a phase-space integral, so that usual sampling techniques can be employed. Notice also that there exist many refinements for those two classes of techniques, such as umbrella sampling [31]. The last and most recent class of methods uses dynamics arising from a switching at a finite rate. This can be done using nonequilibrium dynamics (the so-called fast growth methods) with a suitable exponential reweighting, as introduced by Jarzynski in [15]. Notice that the thermodynamic integration and free energy perturbation methods can be seen respectively as the limits of infinitely slow and fast switching of nonequilibrium dynamics, at least formally. Instead of being imposed a priori, this switching may also arise as the result of an equilibrium sampling, using for example the Adaptive Biasing Force technique [7, 12] or metadynamics [14]. In those cases, the system is progressively forced to leave regions where the sampling of the reaction coordinate has been completed.

It is still a matter of debate which method is the most efficient. While some results show that fast growth methods can be competitive in some situations [11, other studies disagree [19]. The results of [19] indeed indicate that even with the use of efficient path sampling techniques (see also [29, 30, 34]), fast growth methods do not outperform conventional methods such as umbrella sampling or thermodynamic integration (at least in a number of typical cases). However, general conclusions about the efficiency of fast growth methods are still to be drawn, depending on the cases under consideration. We believe that there is room for improvements of this relatively new method (e.g. by optimizing the switching schedule [24]). Let us also mention that this method is straightforward to parallelize and naturally provides with a posteriori error bounds via the central limit theorem, since it involves many independent trajectories.

Most methods to compute free energy differences are well suited to the alchemical transition setting, but do not straightforwardly extend to the reaction coordinate setting. This latter case is the focus throughout this article. In this case, the methods described above require to consider dynamics restricted to the submanifold $\Sigma_{z}$. For computations using Hamiltonian dynamics, we refer for example to [4, 24]. In the stochastic case, thermodynamic integration 
in the reaction coordinate case using projected stochastic dynamics has recently been put on a firm grounding [6, 9]. On the other hand, stochastic nonequilibrium dynamics à la Jarzynski in the reaction coordinate case was, to our knowledge, not studied mathematically. It is the aim of this paper to perform such a study and to present a methodology to compute free energy differences in this framework.

Nonequilibrium computations of free energy differences in the reaction coordinate setting using stochastic dynamics have until now used soft constraints to switch between the initial state centered on the submanifold $\left\{\xi(q)=z_{0}\right\}$ and the final state centered on $\left\{\xi(q)=z_{1}\right\}$. Steered molecular dynamics techniques use for example a penalty term $K(\xi(q)-z)^{2}$ in the energy of the system [22] (with $K$ large) to 'softly' constraint the system to remain close to the submanifold $\{\xi(q)-z=0\}$, and varying the value $z$ from 0 to 1 in a finite time $T$. It is shown in [13] how to use such a biasing potential to exactly compute free energy differences (even for a finite $K$ ), which is of particular interest for experimental studies. From a computational viewpoint however, it is expected that large values of $K$ require small integration time steps. Moreover, it is observed in practice that the statistical fluctuations increase with larger $K$ (see [22]). Instead, we propose to replace the stiff constraining potential $K(\xi(q)-z)^{2}$ by a projection onto the submanifold $\{\xi(q)-z=0\}$. This situation is reminiscent of the case of molecular constraints, that can be enforced using a stiff penalty term, or more elegantly and often more efficiently, using some projection of the dynamics involving Lagrange multipliers. This is the spirit of the well known SHAKE algorithm [26].

We propose a nonequilibrium stochastic dynamics and an equality that allow to compute free energy differences between states defined by different values of a reaction coordinate. The dynamics relies on a projection onto the current submanifold at each time step, and we use the Lagrange multipliers associated with this projection to estimate the free energy difference. More precisely, we use the difference between these Lagrange multipliers and the external forcing term required for the finite time switching (see for example the discretization (31)). The main results of the paper are the Feynman-Kac equality of Theorem 2.2 (which extends the proof of [13] to hard constraints), as well as the associated discretizations (33) and (34).

The method we propose forces the system to pass free energy barriers, and thus enables free energy difference computations for metastable systems. Of course the reliability of the algorithm crucially depends on the choice of the reaction coordinate, which represents the essential degrees of freedom. The reaction coordinate should be rich enough in order to adequately describe the configuration paths of the system from the initial state to the final state. The determination of the essential degrees of freedom of a system is a very important problem, which is not the focus of this work. Thus, in the following, we suppose that a "good" reaction coordinate is given, and we are interested in the computation of free energy differences associated with this reaction coordinate.

Let us also notice that some recent refinements of nonequilibrium dynamics to compute free energy differences, especially path sampling techniques [34] and Interacting Particle Systems approaches 25] (which equilibrate the nonequilibrium dynamics through some birth/death process based on the current work), can be extended to the reaction coordinate setting using the techniques we present here. Moreover, we restrict ourselves to the so-called overdamped Langevin dynamics, but it is possible to extend these results to the usual Langevin dynamics (this is a work in progress).

The paper is organized as follows. In Section 1, the thermodynamic integration setting is outlined in the reaction coordinate case. Section 2 then extends the method to nonequilibrium dynamics. Adapted numerical schemes are proposed in Section 3, and some numerical results 
assessing the correctness of the method are presented in Section 4 For clarity, we present the method in the case of a one-dimensional reaction coordinate and postpone until Appendix $\mathrm{A}$ the proofs and the expressions for the multi-dimensional case.

\section{Equilibrium computation of free energy differences}

The aim of this section is to introduce the definitions of the free energy and the mean force, and to recall how thermodynamic integration is used to compute free energy differences. The computation of the mean force is based on projected stochastic differential equations (SDE). These SDEs will also be used for the discretization of Jarzynski equality in Section 2. This section mainly reviews results of [6].

\subsection{Free energy and mean force}

In the following, we denote by $\mathcal{M} \subset \mathbb{R}^{3 N}$ the configuration space of the system when no parameter $z$ is involved. The state of the system is characterized by the value of a reaction coordinate $\xi: \mathcal{M} \rightarrow[0,1]$. The function $\xi$ is supposed to be smooth and such that $\nabla \xi(q) \neq 0$ for all $q \in \mathcal{M}$. For a given value $z \in[0,1]$, we denote by $\Sigma_{z}$ the submanifold

$$
\Sigma_{z}=\{q \in \mathcal{M}, \xi(q)=z\}
$$

and we assume that $\bigcup_{z \in[0,1]} \Sigma_{z} \subset \mathcal{M}$. For each point $q \in \Sigma_{z}$, we also introduce the orthogonal projection operator $P(q)$ onto the tangent space to $\Sigma_{z}$ at point $q$ defined by:

$$
P(q)=\mathrm{Id}-\frac{\nabla \xi \otimes \nabla \xi}{|\nabla \xi|^{2}}(q),
$$

where $\otimes$ denotes the tensor product. The orthogonal projection operator on the normal space to $\Sigma_{z}$ at point $q$ is defined by $P^{\perp}(q)=\mathrm{Id}-P(q)$.

The free energy is then defined as

$$
F(z)=-\beta^{-1} \ln \left(Z_{z}\right),
$$

with

$$
Z_{z}=\int_{\Sigma_{z}} \exp (-\beta V) d \sigma_{\Sigma_{z}}
$$

where for any submanifold $\Sigma$ of $\mathbb{R}^{3 N}, \sigma_{\Sigma}$ denotes the Lebesgue measure induced on $\Sigma$ as a submanifold of $\mathbb{R}^{3 N}$. The associated Boltzmann probability measure is

$$
d \mu_{\Sigma_{z}}=Z_{z}^{-1} \exp (-\beta V) d \sigma_{\Sigma_{z}} .
$$

Remark 1.1 (On the definition of the free energy). Two comments are in order about formula (5). First, this formula is valid up to an additive constant, which is not important when considering free energy differences. Second, the potential $V$ in (6) may be a potential different from the actual potential seen by the particles. More precisely, if the particles evolve in a potential $V$, the standard definition of the free energy in the physics and chemistry literature is (5) with

$$
Z_{z}=\int \exp (-\beta V) \delta_{\xi(q)-z},
$$


where $\delta_{\xi(q)-z}$ is a measure supported by $\Sigma_{z}$ and defined by: for all test functions $\phi$,

$$
\int \phi(q) \delta_{\xi(q)-z}=\int_{\Sigma_{z}} \phi|\nabla \xi|^{-1} d \sigma_{\Sigma_{z}}
$$

This amounts to considering (5)-(6) with $V$ replaced by an effective potential $V+\beta^{-1} \ln |\nabla \xi|$ (see Remark A.1 for the case of a multi-dimensional constraint). Since the results we present in this paper hold irrespective of the physical signification of the potential $V$, we may assume without loss of mathematical generality that the free energy is indeed given by (5)-(6). Let us emphasize that, in practice, the cumbersome computation of the gradient of the additional term $\beta^{-1} \ln |\nabla \xi|$ in the modified potential (which intervenes in the projected SDEs we use, see (27)-(28) or (29)-(30)) can be avoided resorting to some finite differences, as explained in [6].

Using the co-area formula (see (42) and Proposition A.2 for a proof in the multi-dimensional case), it is possible to derive the following expression of the derivative of the free energy $F$ with respect to $z$ (the so-called mean force) (see [21, 27]):

$$
F^{\prime}(z)=Z_{z}^{-1} \int_{\Sigma_{z}} \frac{\nabla \xi}{|\nabla \xi|^{2}} \cdot\left(\nabla V+\beta^{-1} H\right) \exp (-\beta V) d \sigma_{\Sigma_{z}}
$$

where

$$
H=-\nabla \cdot\left(\frac{\nabla \xi}{|\nabla \xi|}\right) \frac{\nabla \xi}{|\nabla \xi|}
$$

is the mean curvature vector field of the surface $\Sigma_{z}$. The free energy can thus be expressed as an average with respect to $\mu_{\Sigma_{z}}$ :

$$
F^{\prime}(z)=\int_{\Sigma_{z}} f(q) d \mu_{\Sigma_{z}}(q)
$$

where $f$ is the local mean force defined by:

$$
f=\frac{\nabla \xi}{|\nabla \xi|^{2}} \cdot\left(\nabla V+\beta^{-1} H\right)
$$

In next section, we will explain how it is possible to compute this average with respect to $\mu_{\Sigma_{z}}$, without explicitly computing $f$, by using projected SDEs. This avoids in particular the computation of the mean curvature vector $H$ which involves second-order derivatives of $\xi$.

The principle of thermodynamic integration is to recast the free energy difference

$$
\Delta F(z)=F(z)-F(0)
$$

between two reaction coordinates 0 and $z$ as an integral over the mean force:

$$
\Delta F(z)=\int_{0}^{z} F^{\prime}(y) d y .
$$

Therefore, in practice, thermodynamic integration computation of free-energy is as follows. First, the free energy difference $\Delta F(z)$ is estimated using quadrature formulae for the integral in (13), such as for example a Gauss-Lobatto scheme:

$$
\Delta F(z) \simeq \sum_{i=0}^{K} \omega_{i} F^{\prime}\left(y_{i}\right)
$$


where the points $\left\{y_{0}, y_{1}, \ldots, y_{K}\right\}$ are in $[0, z]$ and $\left\{\omega_{0}, \omega_{1}, \ldots, \omega_{K}\right\}$ are their associated weights. Second, the derivatives $F^{\prime}\left(y_{i}\right)$ are computed as canonical averages over the submanifolds $\Sigma_{y_{i}}$, using projected SDEs (see next section).

To obtain a free-energy profile (and not only a free-energy difference for a fixed final state), it is possible to approximate the function $\Delta F(z)$ on the interval $[0,1]$ by a polynomial. This can be done for example by interpolating the derivative $F^{\prime}$ by splines, and integrating the resulting function (consistently with the normalization $\Delta F(0)=0$ ).

\subsection{Projected stochastic differential equations}

In this section, we explain how to compute the mean force $F^{\prime}(z)$ defined by (8) using projected SDEs, for a fixed parameter $z$. We consider the solution $Q_{t}$ to the following SDE:

$$
\left\{\begin{array}{l}
Q_{0} \in \Sigma_{z}, \\
d Q_{t}=-P\left(Q_{t}\right) \nabla V\left(Q_{t}\right) d t+\sqrt{2 \beta^{-1}} P\left(Q_{t}\right) \circ d B_{t},
\end{array}\right.
$$

where $B_{t}$ is the standard $3 N$-dimensional Brownian motion and o denotes the Stratonovich product. It is possible (see [6]) to check that $\mu_{\Sigma_{z}}$ is an invariant probability measure associated with the SDE (14). Under suitable assumptions, which we assume in the rest of the section, on the potential $V$ and the surface $\Sigma_{z}$, the process $Q_{t}$ is ergodic with respect to $\mu_{\Sigma_{z}}$. Moreover, the SDE (14) can be rewritten in the following way:

$$
d Q_{t}=-\nabla V\left(Q_{t}\right) d t+\sqrt{2 \beta^{-1}} d B_{t}+\nabla \xi\left(Q_{t}\right) d \Lambda_{t},
$$

where $\Lambda_{t}$ is a real valued process, which can be interpreted as the Lagrange multiplier associated with the constraint $\xi\left(Q_{t}\right)=z$ (see the discretization in Section 3.1). This process can be decomposed into two parts:

$$
d \Lambda_{t}=d \Lambda_{t}^{\mathrm{m}}+d \Lambda_{t}^{\mathrm{f}} .
$$

The so-called martingale 1 part $\Lambda_{t}^{\mathrm{m}}$ (whose fluctuation is of order $\sqrt{\Delta t}$ over a timestep $\Delta t$ ) is

$$
d \Lambda_{t}^{\mathrm{m}}=-\sqrt{2 \beta^{-1}} \frac{\nabla \xi}{|\nabla \xi|^{2}}\left(Q_{t}\right) \cdot d B_{t}
$$

where implicitly denotes the Itô product. The so-called bounded variation part $\Lambda_{t}^{\mathrm{f}}$ (whose fluctuation is of order $\Delta t$ over a timestep $\Delta t$ ) is

$$
d \Lambda_{t}^{\mathrm{f}}=\frac{\nabla \xi}{|\nabla \xi|^{2}}\left(Q_{t}\right) \cdot \nabla V\left(Q_{t}\right) d t+\beta^{-1} \frac{\nabla \xi}{|\nabla \xi|^{2}}\left(Q_{t}\right) \cdot H\left(Q_{t}\right) d t=f\left(Q_{t}\right) d t
$$

$f$ being the local mean force defined above by (11). Thus, since $Q_{t}$ is ergodic with respect to $\mu_{\Sigma_{z}}$ the mean force can be obtained as a mean over the Lagrange multiplier $\Lambda_{t}$ :

Proposition 1.2. The mean force is given by:

$$
F^{\prime}(z)=\lim _{T \rightarrow \infty} \frac{1}{T} \int_{0}^{T} d \Lambda_{t}=\lim _{T \rightarrow \infty} \frac{1}{T} \int_{0}^{T} d \Lambda_{t}^{\mathrm{f}} .
$$

\footnotetext{
${ }^{1}$ For our purposes, it is enough to think of a martingale as an Itô integral with respect to the Brownian motion $\left(B_{t}\right)_{t \geq 0}$.
} 
Notice that the martingale part $d \Lambda_{t}^{\mathrm{m}}$, which has the largest fluctuations, has zero mean. In order to reduce the variance, it is thus numerically convenient to perform the mean over the bounded variation part $d \Lambda_{t}^{\mathrm{f}}$ rather than over the whole Lagrange multiplier $d \Lambda_{t}$ (see Section 3).

We refer to 6] for a proof of Proposition 1.2, as well as for formulae involving higher dimensional reaction coordinates. Such ideas have been used for a long time in the framework of Hamiltonian dynamics (see [21, 27]).

The interest of Equation (19) is that the SDE (15) can be very naturally discretized as explained in Section 3.1 below. Then, the average over a discretized trajectory of the process $\Lambda_{t}$ converges to $F^{\prime}(z)$. This is particularly convenient for numerical purposes since it does not ask for explicitly computing the local force $f$. For further details, we refer to 6 and to Section 3.1. In next section, we use these ideas for the computation of the free energy difference given through the Jarzynski equality.

\section{Nonequilibrium stochastic methods in the reaction coordi- nate case}

As opposed to quasistatic methods where the free energy difference between an initial state and a final state is expressed by (13), in nonequilibrium methods, the free energy difference is expressed using a Feynman-Kac average over nonequilibrium paths [15, 13, 25]

$$
\Delta F(1)=F(1)-F(0)=-\beta^{-1} \ln \mathbb{E}\left(\mathrm{e}^{-\beta \mathcal{W}(T)}\right),
$$

where $\mathcal{W}(T)$ denotes the total work exerted along a nonequilibrium path $\left(Q_{t}, z(t)\right)_{t \in[0, T]}$, with $z(0)=0$ and $z(T)=1$.

We wish here to extend the Feynman-Kac formula derived in [13] for a parameter $z$ which appears only in the potential $V$, to the reaction coordinate case, where $z$ labels submanifolds $\Sigma_{z}$ (defined by Equation (3)) of the state space. To this end, we need to make precise the evolution of the constraints.

We consider a $\mathcal{C}^{1}$ path $z:[0, T] \rightarrow[0,1]$ of values of the reaction coordinate $\xi$, with $z(0)=0$, and $z(T)=1$. Recall that the associated family of submanifolds of admissible configurations is denoted by

$$
\Sigma_{z(t)}=\{q \in \mathcal{M}, \xi(q)=z(t)\},
$$

and that the associated Boltzmann probability measures are

$$
d \mu_{\Sigma_{z(t)}}=Z_{z(t)}^{-1} \exp (-\beta V) d \sigma_{\Sigma_{z(t)}} .
$$

We construct a diffusion $\left(Q_{t}\right)_{t \in[0, T]}$ so that $Q_{t} \in \Sigma_{z(t)}$ for all $t \in[0, T]$ and $\left(Q_{t}\right)_{t \in[0, T]}$ satisfies the following properties (see Section 2.1 for a more rigorous formulation):

- $Q_{0} \sim \mu_{\Sigma_{z(0)}}$,

- For all $t \in[0, T], Q_{t+d t}$ is the orthogonal projection on $\Sigma_{z(t+d t)}$ of the position obtained by the unconstrained displacement: $Q_{t}-\nabla V\left(Q_{t}\right) d t+\sqrt{2 \beta^{-1}} d B_{t}$.

To each realization of this process, a work $\mathcal{W}(t)$ can be associated as

$$
\mathcal{W}(t)=\int_{0}^{t} f\left(Q_{s}\right) z^{\prime}(s) d s
$$


where $f$ is the local mean force defined above by (11). Then, we prove that the FeynmanKac formula (20) holds for the free energy $F$ associated with the reaction coordinate and defined by (5). Notice that, at least formally, in the limit of an infinitely slow switching from $z(0)=0$ to $z(T)=1$, Formula (20) corresponds to the thermodynamic integration formula (13). Formula (20) enables the computation of free energy difference at arbitrary rates, through a correction consisting in a reweighting of the nonequilibrium paths.

The rest of this section is organized as follows. In Section 2.1, we make precise the process $Q_{t}$ we consider. Then, in Section 2.2, we state the Feynman-Kac formula (20) for a one-dimensional reaction coordinate. We recall that the formulae for the general case involving higher dimensional reaction coordinates, as well as the main proofs, are presented in Appendix $\mathrm{A}$.

\subsection{The nonequilibrium projected stochastic dynamics}

The considered diffusion reads, in the Stratonovich setting:

$$
\left\{\begin{array}{l}
Q_{0} \sim \mu_{\Sigma_{z(0)}}, \\
d Q_{t}=-P\left(Q_{t}\right) \nabla V\left(Q_{t}\right) d t+\sqrt{2 \beta^{-1}} P\left(Q_{t}\right) \circ d B_{t}+\nabla \xi\left(Q_{t}\right) d \Lambda_{t}^{\mathrm{ext}}, \\
d \Lambda_{t}^{\mathrm{ext}}=\frac{z^{\prime}(t)}{\left|\nabla \xi\left(Q_{t}\right)\right|^{2}} d t .
\end{array}\right.
$$

With a view to the discretization of $Q_{t}$, let us notice that $Q_{t}$ can be characterized by the following property:

Proposition 2.1. The process $Q_{t}$ solution to (21) is the only Itô process satisfying for some real-valued adapted Itô process $\left(\Lambda_{t}\right)_{t \in[0, T]}$ :

$$
\begin{cases}Q_{0} & \sim \mu_{\Sigma_{z(0)}} \\ d Q_{t} & =-\nabla V\left(Q_{t}\right) d t+\sqrt{2 \beta^{-1}} d B_{t}+\nabla \xi\left(Q_{t}\right) d \Lambda_{t} \\ \xi\left(Q_{t}\right) & =z(t) .\end{cases}
$$

Moreover, the process $\left(\Lambda_{t}\right)_{t \in[0, T]}$ can be decomposed as

$$
\Lambda_{t}=\Lambda_{t}^{\mathrm{m}}+\Lambda_{t}^{\mathrm{f}}+\Lambda_{t}^{\mathrm{ext}}
$$

with the martingale part

$$
d \Lambda_{t}^{\mathrm{m}}=-\sqrt{2 \beta^{-1}} \frac{\nabla \xi}{|\nabla \xi|^{2}}\left(Q_{t}\right) \cdot d B_{t},
$$

the local force part (see (11) for the definition of $f$ )

$$
d \Lambda_{t}^{\mathrm{f}}=\frac{\nabla \xi}{|\nabla \xi|^{2}}\left(Q_{t}\right) \cdot\left(\nabla V\left(Q_{t}\right) d t+\beta^{-1} H\left(Q_{t}\right)\right) d t=f\left(Q_{t}\right) d t
$$

and the external forcing (or switching) term

$$
d \Lambda_{t}^{\mathrm{ext}}=\frac{z^{\prime}(t)}{\left|\nabla \xi\left(Q_{t}\right)\right|^{2}} d t
$$


The proof of Proposition 2.1 is easy and consists in computing $d \xi\left(Q_{t}\right)$ by Itô's calculus and identifying the bounded variation and the martingale parts of the stochastic processes.

The difference with the projected stochastic differential equation (14) considered in the thermodynamic integration setting is that the out-of-equilibrium evolution of the constraints $z(t)$ creates a drift $\nabla \xi\left(Q_{t}\right) d \Lambda_{t}^{\text {ext }}$ along the reaction coordinate. This drift can be interpreted as an external forcing required for the switching to take place at a finite rate, and must be subtracted from the Lagrange multiplier $\Lambda_{t}$ in order to obtain a correct expression for the work $\mathcal{W}(t)$ involved in the Feynman-Kac fluctuation equality (see Equations (31) and (33) below). This correction is quantitatively important when the switching is not slow.

\subsection{The Feynman-Kac fluctuation equality}

Let us define the nonequilibrium work exerted on the diffusion (21) by:

$$
\mathcal{W}(t)=\int_{0}^{t} f\left(Q_{s}\right) z^{\prime}(s) d s
$$

where $f$ is the local mean force defined above by (11). In practice, the nonequilibrium work $\mathcal{W}(t)$ can be computed by using the local force part $d \Lambda_{t}^{\mathrm{f}}$ (see (23) ), as in the thermodynamic integration method (see (19) ). Thus, the formula we use to compute $\mathcal{W}(t)$ is rather:

$$
\mathcal{W}(t)=\int_{0}^{t} z^{\prime}(s) d \Lambda_{s}^{\mathrm{f}}
$$

since $\Lambda_{t}^{\mathrm{f}}$ can be obtained by a natural numerical scheme (see Section 3 ), avoiding the cumbersome computations of the mean curvature vector $H$ in the expression of $f$ (as already explained in Section 1.1).

We can now state the generalization of the Jarzynski nonequilibrium equality to the case when the switching is parameterized by a reaction coordinate.

Theorem 2.2 (Feynman-Kac fluctuation equality). For any test function $\varphi$ and $\forall t \in[0, T]$, it holds

$$
\frac{Z_{z(t)}}{Z_{z(0)}} \int_{\Sigma_{z(t)}} \varphi d \mu_{\Sigma_{z(t)}}=\mathbb{E}\left(\varphi\left(Q_{t}\right) \mathrm{e}^{-\beta \mathcal{W}(t)}\right) .
$$

In particular, we have the work fluctuation identity: $\forall t \in[0, T]$,

$$
\Delta F(z(t))=F(z(t))-F(z(0))=-\beta^{-1} \ln \left(\mathbb{E}\left(\mathrm{e}^{-\beta \mathcal{W}(t)}\right)\right) .
$$

As in the alchemical case [13], the proof follows from a Feynman-Kac formula. The proof of this theorem is presented in the general multi-dimensional case in Appendix $\mathrm{A}$ (see Theorem A.5).

\section{Discretization of the dynamics}

The main interest of the above formulae (13)-(19) and (25) $-(26)$ is that they admit natural time discretizations. The principle is to use a predictor-corrector scheme for the associated dynamics (14) and (21), and to use the Lagrange multiplier $\Lambda_{t}$ to compute the local mean force $f$. 
Section 3.1 is mainly a review of the results of [6] and presents this idea in the context of thermodynamic integration. Then, we extend the method to the case of evolving constraints in Section 3.2 .

\subsection{Discretization of the projected diffusion}

For the projected SDE (15) onto a submanifold $\Sigma_{z}=\{\xi(q)-z=0\}$, two discretizations of the dynamics, extending the usual Euler-Maruyama scheme, are proposed in [6]. These numerical schemes for constrained Brownian dynamics are in the spirit of the so-called RATTLE [2] and SHAKE [26] algorithms classical used for constrained Hamiltonian dynamics, and also related with the algorithms proposed in [32, 1, 20].

The first one is:

$$
\left\{\begin{array}{l}
Q_{n+1}=Q_{n}-\nabla V\left(Q_{n}\right) \Delta t+\sqrt{2 \Delta t \beta^{-1}} U_{n}+\Delta \Lambda_{n+1} \nabla \xi\left(Q_{n+1}\right) \\
\text { where } \Delta \Lambda_{n+1} \text { is such that } \xi\left(Q_{n+1}\right)=z
\end{array}\right.
$$

where $\Delta t$ is the time step and $U^{n}$ is a $3 N$-dimensional standard Gaussian random vector. Notice that (27) admits a natural variational interpretation, since $Q_{n+1}$ can be seen as the closest point on the submanifold $\Sigma_{z}$ to the predicted position $Q_{n}-\nabla V\left(Q_{n}\right) \Delta t+\sqrt{2 \Delta t \beta^{-1}} U_{n}$. The real $\Delta \Lambda_{n+1}$ is then the Lagrange multiplier associated with the constraint $\xi\left(Q_{n+1}\right)=z$.

Another possible discretization of (15) is

$$
\left\{\begin{array}{l}
Q_{n+1}=Q_{n}-\nabla V\left(Q_{n}\right) \Delta t+\sqrt{2 \Delta t \beta^{-1}} U_{n}+\Delta \Lambda_{n+1} \nabla \xi\left(Q_{n}\right) \\
\text { where } \Delta \Lambda_{n+1} \text { is such that } \xi\left(Q_{n+1}\right)=z
\end{array}\right.
$$

Although this scheme is not naturally associated with a variational principle, it may be more practical since its formulation is more explicit. Notice also that we use the same notation $\Delta \Lambda_{n}$ for the Lagrange multipliers for both (27) and (28) (and later for (29) and (30)), since all the formulas we state in terms of $\Delta \Lambda_{n}$ are verified whatever the constrained dynamics.

To solve Equation (27), classical methods for optimization problems with constraints can be used. We refer to [10] for a presentation of the classical Uzawa algorithm, and to [3] for more advanced methods. Problem (28) can be solved using classical methods for nonlinear problems, such as the Newton method (see [3]). We also refer to Chapter 7 of [18 where similar problems are discussed, for the classical RATTLE and SHAKE schemes used for Hamiltonian dynamics with constraints.

Both schemes are consistent (the discretization error goes to 0 when the time step $\Delta t$ goes to 0) with the projected diffusion (15) (see [6]). Accordingly, $\Delta \Lambda_{n+1}$ is a consistent discretization of $\int_{t_{n}}^{t_{n+1}} d \Lambda_{t}$ and therefore, it can be proven [6]:

$$
\lim _{T \rightarrow \infty} \lim _{\Delta t \rightarrow 0} \frac{1}{T} \sum_{n=1}^{T / \Delta t} \Delta \Lambda_{n}=F^{\prime}(z)
$$

which is the discrete counterpart of the trajectory average (19). In [6], a variance reduction technique is proposed, which consists in extracting the bounded variation part $\Delta \Lambda_{n}^{\mathrm{f}}$ of $\Delta \Lambda_{n}$ (resorting locally to reversed Brownian increments). We give some details of an adaptation of this method for evolving constraints in next section. 


\subsection{Discretization with evolving constraints}

When nonequilibrium dynamics are considered, the constraint is stated as $\xi\left(Q_{t}\right)=z(t)$. The reaction coordinate path is first discretized as $\left\{z(0), \ldots, z\left(t_{N_{T}}\right)\right\}$ where $N_{T}$ is the number of timesteps. For example, equal time increments can be used, in which case $\Delta t=\frac{T}{N_{T}}$ and $t_{n}=n \Delta t$ (we refer to Remark 3.1 below for some refinements). The initial conditions $Q_{0}$ are sampled according to $\mu_{\Sigma_{0}}$. A way to do that is to subsample a long trajectory of the projected SDE on $\Sigma_{0}$ (using the schemes (27) or (28)).

The projected SDE on evolving constraints (21) is then discretized with the scheme (27) or (28), taking into account the evolution of the constraint:

$$
\left\{\begin{array}{l}
Q_{n+1}=Q_{n}-\nabla V\left(Q_{n}\right) \Delta t+\sqrt{2 \Delta t \beta^{-1}} U_{n}+\Delta \Lambda_{n+1} \nabla \xi\left(Q_{n+1}\right), \\
\text { where } \Delta \Lambda_{n+1} \text { is such that } \xi\left(Q_{n+1}\right)=z\left(t_{n+1}\right),
\end{array}\right.
$$

or

$$
\left\{\begin{array}{l}
Q_{n+1}=Q_{n}-\nabla V\left(Q_{n}\right) \Delta t+\sqrt{2 \Delta t \beta^{-1}} U_{n}+\Delta \Lambda_{n+1} \nabla \xi\left(Q_{n}\right) \\
\text { where } \Delta \Lambda_{n+1} \text { is such that } \xi\left(Q_{n+1}\right)=z\left(t_{n+1}\right)
\end{array}\right.
$$

It remains to extract the force part $\Delta \Lambda_{n+1}^{\mathrm{f}}$ from the discretized Lagrange multiplier $\Delta \Lambda_{n+1}$ (consistently with (22)). We propose two methods. First, this can be done by simply subtracting the drift and the martingale part

$$
\Delta \Lambda_{n+1}^{\mathrm{f}}=\Delta \Lambda_{n+1}-\frac{z\left(t_{n+1}\right)-z\left(t_{n}\right)}{\left|\nabla \xi\left(Q_{n}\right)\right|^{2}}+\sqrt{2 \Delta t \beta^{-1}} \frac{\nabla \xi\left(Q_{n}\right)}{\left|\nabla \xi\left(Q_{n}\right)\right|^{2}} \cdot U_{n} .
$$

Another possibility in the spirit of the variance reduction techniques used in [6] can also be used. Consider the following coupled dynamic with locally time-reversed constraint evolution (written here for the scheme (29)):

$$
Q_{n+1}^{\mathrm{R}}=Q_{n}-\nabla V\left(Q_{n}\right) \Delta t-\sqrt{2 \Delta t \beta^{-1}} U_{n}+\Delta \Lambda_{n+1}^{\mathrm{R}} \nabla \xi\left(Q_{n+1}^{\mathrm{R}}\right),
$$

with $\Delta \Lambda_{n+1}^{\mathrm{R}}$ such that:

$$
\frac{1}{2}\left(\xi\left(Q_{n+1}^{\mathrm{R}}\right)+\xi\left(Q_{n+1}\right)\right)=\xi\left(Q_{n}\right) .
$$

The position $Q_{n+1}^{\mathrm{R}}$ is computed as $Q_{n+1}$ in (29), but with a projection on $\Sigma_{2 \xi\left(Q_{n}\right)-\xi\left(Q_{n+1}\right)}$ instead of $\Sigma_{z\left(t_{n+1}\right)}$, and using the Brownian increment $-\sqrt{\Delta t} U_{n}$ instead of $\sqrt{\Delta t} U_{n}$. Notice that in case of a constant increment for the constraints, we have $\xi\left(Q_{n+1}^{\mathrm{R}}\right)=2 \xi\left(Q_{n}\right)-\xi\left(Q_{n+1}\right)=$ $z\left(t_{n-1}\right)$. The force part $\Delta \Lambda_{n+1}^{\mathrm{f}}$ is then obtained through

$$
\Delta \Lambda_{n+1}^{\mathrm{f}}=\frac{1}{2}\left(\Delta \Lambda_{n+1}+\Delta \Lambda_{n+1}^{\mathrm{R}}\right)
$$

which can be shown to be a consistent time discretization of $\int_{t_{n}}^{t_{n+1}} d \Lambda_{t}^{\mathrm{f}}$.

\subsection{Computation of free energy using a Feynman-Kac equality}

The consistent discretization of $Q_{t}$, and more precisely of $\int_{t_{n}}^{t_{n+1}} d \Lambda_{t}^{\mathrm{f}}$, we have obtained in the previous section can now be used to approximate the work $\mathcal{W}(t)$ defined by (25) by

$$
\left\{\begin{array}{l}
\mathcal{W}_{0}=0 \\
\mathcal{W}_{n+1}=\mathcal{W}_{n}+\frac{z\left(t_{n+1}\right)-z\left(t_{n}\right)}{t_{n+1}-t_{n}} \Delta \Lambda_{n+1}^{\mathrm{f}},
\end{array}\right.
$$


using either the dynamics (29) or (30), and the local force part of the Lagrange multiplier computed by (31) or (32). Averaging over $M$ independent realizations (the corresponding works being labeled by an upper index $1 \leq m \leq M)$, an estimator of the free energy difference $\Delta F(z(T))$ is, using Theorem 2.2 ,

$$
\widehat{\Delta F}(z(T))=-\beta^{-1} \ln \left(\frac{1}{M} \sum_{m=1}^{M} \mathrm{e}^{-\beta \mathcal{W}_{N_{T}}^{m}}\right) .
$$

The estimator $\widehat{\Delta F}(z(T))$ converges to $\Delta F(z(T))$ as $\Delta t \rightarrow 0$ and $M \rightarrow+\infty$. It is clear that the estimation of $\Delta F(z(T))$ by (34) is straightforward to parallelize since the $\left(\mathcal{W}_{N_{T}}^{m}\right)_{1 \leq m \leq M}$ are independent.

Notice that, even in the limit $\Delta t \rightarrow 0, \widehat{\Delta F}(z(T))$ is a biased estimator. Indeed, $\exp (-\beta \widehat{\Delta F}(z(T)))$ is an unbiased estimator of $\exp (-\beta \Delta F(z(T)))$, and therefore, using the concavity of $\ln , \mathbb{E}(\widehat{\Delta F}(z(T))) \geq \Delta F(z(T))$. Recent works propose corrections to this systematic bias using asymptotic expansions in the limit $M \rightarrow+\infty$ (see for instance [24, 36]).

Remark 3.1 (On practical implementation). Notice that it may be useful to adaptively refine the time step over each stochastic trajectories, using for example the work evolution rate $\left(\mathcal{W}_{n}-\right.$ $\left.\mathcal{W}_{n-1}\right)_{n \geq 1}$ as a refinement criterion.

As noticed in [24], it is also possible to optimize the evolution of the constraint $z(t)$, for example by minimizing the variance of the results obtained for a priori schedules for the evolving constraint on a small set of preliminary runs.

\section{Numerical results}

We present in this section some illustrations of the algorithm we have described above to compute free energy differences through nonequilibrium paths. In Section 4.1, a two-dimensional toy potential $V$ is used, for which we can compare the results with analytical profiles. A more realistic test case in Section 4.2 demonstrates the ability of the method to compute free energy profiles in presence of a free energy barrier.

Our aim in this section is not to compare the numerical efficiency of the thermodynamic integration method presented in Section 1 (or any other method) with nonequilibrium computations, since it is difficult to draw general conclusions about such comparisons. However, we compare on a simple example in Section 4.1, the numerical efficiency of out-of-equilibrium computations using a few long trajectories or many short trajectories, at a fixed computational cost.

\subsection{A two-dimensional toy problem}

We consider the two-dimensional potential introduced in 33

$$
V(x, y)=\cos (2 \pi x)\left(1+d_{1} y\right)+d_{2} y^{2},
$$

where $d_{1}$ and $d_{2}$ are two positive constants. Some corresponding Boltzmann-Gibbs probability densities are depicted in Figure 1.

We want to compute the free energy difference profile between the initial state $x=x_{0}=$ -0.5 and the transition state $x=x_{1}=0$. Notice that the saddle point is $\left(x_{1}, y_{1}\right)=(0,0)$ for 

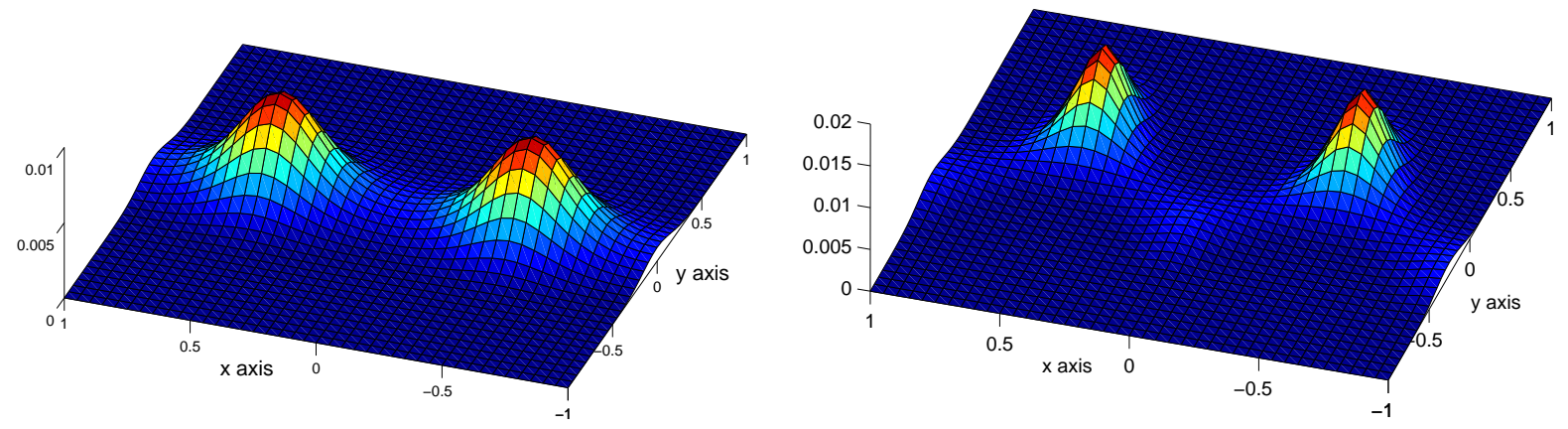

Figure 1: Plot of some probability densities corresponding to the potential (35) for $\beta=1$, $d_{2}=2 \pi^{2}$, and $d_{1}=0$ on the left or $d_{1}=10$ on the right.

$d_{1}=0$, but is increasingly shifted toward lower values of $y_{1}$ as $d_{1}$ increases. We parameterize the transition along the $x$-axis, either with the reaction coordinate

$$
\xi(x, y)=\frac{x-x_{0}}{x_{1}-x_{0}}
$$

or with the reaction coordinate $(n \geq 2)$

$$
\eta_{n}(x, y)=\frac{1}{2^{n}-1}\left[\left(1+\frac{x-x_{0}}{x_{1}-x_{0}}\right)^{n}-1\right]
$$

For these reaction coordinates, the initial state (resp. the transition state) corresponds to a value of the reaction coordinate $z=0$ (resp. $z=1$ ). The analytical expression of the free energy difference that we consider here is, for a reaction coordinate $\nu(x, y)$ (such as $\xi$ or $\eta_{n}$ defined above)

$$
\Delta F_{\nu}(z)=-\beta^{-1} \ln \left(\frac{\int \mathrm{e}^{-\beta V(x, y)} \delta_{\nu(x, y)-z}}{\int \mathrm{e}^{-\beta V(x, y)} \delta_{\nu(x, y)}}\right),
$$

where the distribution $\delta_{\nu(x, y)-z}$ is defined in Remark 1.1] above. Notice that even though the initial state $\Sigma_{0}=\{x=-0.5\}$ and the final state $\Sigma_{1}=\{x=0\}$ are the same for the reaction coordinates $\xi$ and $\eta_{n}$, the associated free energy differences differ. This is due to the fact that $\nabla \xi \neq \nabla \eta_{n}$, and therefore $\delta_{\xi(x, y)-z} \neq \delta_{\eta_{n}(x, y)-z}$. More precisely,

$$
\Delta F_{\xi}(z)=-\cos \left(2 \pi x_{0}\right)+\cos \left(2 \pi x_{\xi}(z)\right)+\frac{\left(d_{1}\right)^{2}}{4 d_{2}}\left(\cos ^{2}\left(2 \pi x_{0}\right)-\cos ^{2}\left(2 \pi x_{\xi}(z)\right)\right)
$$

with

$$
x_{\xi}(z)=x_{0}+z\left(x_{1}-x_{0}\right),
$$



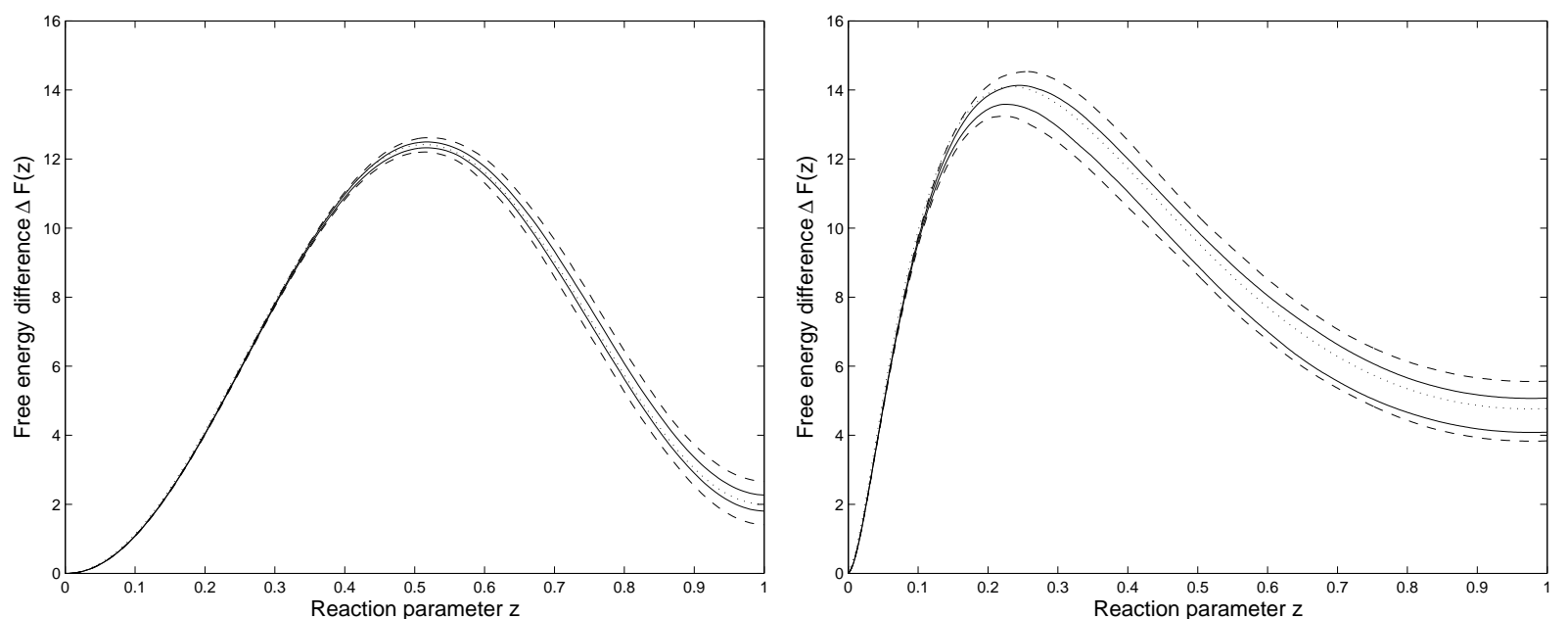

Figure 2: Free energy profiles using the potential (35) with $\beta=1, d_{1}=30$ and $d_{2}=2 \pi^{2}$, and the reaction coordinate (36) on the left, or the reaction coordinate (37) with $n=5$ on the right. Analytical reference profiles are in dotted lines. The dashed lines (resp. the solid lines) represent the upper and lower bound of the $95 \%$ confidence interval (obtained over 100 independent realizations) for nonequilibrium computations with $M=10^{3}$ replicas (resp. with $M=10^{4}$ replicas). The switching time is $T=1$ and the time step is $\Delta t=0.005$ on the left and $\Delta t=0.0025$ on the right.

and

$$
\begin{aligned}
\Delta F_{\eta_{n}}(z)= & -\cos \left(2 \pi x_{0}\right)+\cos \left(2 \pi x_{\eta_{n}}(z)\right)+\frac{\left(d_{1}\right)^{2}}{4 d_{2}}\left(\cos ^{2}\left(2 \pi x_{0}\right)-\cos ^{2}\left(2 \pi x_{\eta_{n}}(z)\right)\right) \\
& +\frac{n-1}{\beta} \ln \left(1+\frac{x_{\eta_{n}}(z)-x_{0}}{x_{1}-x_{0}}\right),
\end{aligned}
$$

with

$$
\left.x_{\eta_{n}}(z)=x_{0}+\left(\left(2^{n}-1\right) z+1\right)^{1 / n}-1\right)\left(x_{1}-x_{0}\right) .
$$

Free energy profiles for the two reaction coordinates considered here can then be computed using the discretization proposed in Section 3.3. Averaging over several realizations, error estimates can be proposed: in particular, the standard deviation can be computed for all intermediate points $z \in[0,1]$, so that, for all values $z$, a confidence interval around the empirical mean can be proposed. We represent on Figure 2 the analytical profiles, and the lower and upper bounds of the $95 \%$ confidence interval for $M=10^{3}$ and $M=10^{4}$, using here and henceforth a linear schedule: $z(t)=t / T$. The initial conditions are created by subsampling every 100 timesteps a trajectory constrained to remain on the initial submanifold $\Sigma_{0}$. As announced above, the profiles obtained with $\eta_{n}$ and $\xi$ are not exactly the same, though the general shape is preserved. These figures also show that the variance increases with $z$. Therefore, to further test the convergence of the method, it is enough here to characterize the convergence of the value for the end point at $z=1$.

We study the convergence of the end value $\Delta F(1)$ computed with the out-of-equilibrium dynamics with respect to the number of replicas $M$ and the time step $\Delta t$, using the reaction coordinate (36) as an example. The results are presented in Table 1 . The time step $\Delta t$ 


\begin{tabular}{|ccc|cc|}
\hline \hline$\Delta t$ & $T$ & $M$ & \multicolumn{2}{|c|}{$\widehat{\Delta F(z(T))}$} \\
\hline 0.001 & 1 & $10^{3}$ & 2.056 & $(0.274)$ \\
0.0025 & 1 & $10^{3}$ & 2.033 & $(0.259)$ \\
0.005 & 1 & $10^{3}$ & 2.076 & $(0.286)$ \\
0.01 & 1 & $10^{3}$ & 2.073 & $(0.278)$ \\
\hline 0.005 & 1 & $10^{3}$ & 2.076 & $(0.286)$ \\
0.005 & 1 & $10^{4}$ & 2.014 & $(0.116)$ \\
0.005 & 1 & $10^{5}$ & 2.001 & $(0.045)$ \\
\hline \hline
\end{tabular}

\begin{tabular}{|ccc|c|}
\hline \hline$\Delta t$ & $T$ & $M$ & \multicolumn{2}{|c|}{$\widehat{\Delta F(z(T))}$} \\
\hline 0.005 & 1 & $10^{4}$ & $2.014(0.116)$ \\
0.005 & 10 & $10^{3}$ & $1.999(0.029)$ \\
0.005 & 100 & $10^{2}$ & $2.001(0.025)$ \\
0.005 & 1000 & $10^{1}$ & $1.997(0.022)$ \\
\hline \hline
\end{tabular}

Table 1: Free energy differences $\Delta F(1)$ obtained by nonequilibrium computations for the reaction coordinate (36) with $\beta=1, d_{1}=1$ and $d_{2}=30$. The results are presented as follows: $\mathbb{E}(\widehat{\Delta F}(z(T))) \quad(\sqrt{\operatorname{Var}(\widehat{\Delta F}(z(T)))})$ (the estimates of these quantities are obtained by averages over 100 independent runs). The exact value is $\Delta F(1)=2$.

does not seem to have any noticeable influence on the final result, as long as it remains in a reasonable range. As expected, the error gets smaller as $M$ increases.

In Table 1, we also show that, in this particular case, for a fixed computational cost and provided that the switching time is large enough 2 , computing many short trajectories is as efficient as computing a few longer ones (the mean and the variance are essentially unchanged). This conclusion also holds for the more realistic test case presented in next section. The computation of many trajectories can be straightforwardly and very efficiently parallelized.

We finally mention that we are able to exhibit the bias of the Jarzynski estimator in this particular case (see Section 3.3 and [36]). We observe that the estimator $\widehat{\Delta F}(z(T))$ is generally greater than $\Delta F(z(T))$. More precisely, averaging over $10^{4}$ realizations, with the parameters $T=1$ and $\Delta t=0.005$, we obtain the following $95 \%$ confidence intervals for $\widehat{\Delta F}(z(T))$, for various values of $M: \widehat{\Delta F}(z(T))=2.0576 \pm 0.0059$ for $M=10^{3}, \widehat{\Delta F}(z(T))=2.0095 \pm 0.0026$ for $M=10^{4}$, and $\widehat{\Delta F}(z(T))=2.00075 \pm 0.0010$ for $M=10^{5}$. As expected, the bias goes to zero when $M \rightarrow \infty$.

\subsection{Model system for conformational changes influenced by solvation}

We consider a system composed of $N$ particles in a periodic box of side length $l$, interacting through the purely repulsive WCA pair potential [8, 28]:

$$
V_{\mathrm{WCA}}(r)=\left\{\begin{array}{cl}
4 \epsilon\left[\left(\frac{\sigma}{r}\right)^{12}-\left(\frac{\sigma}{r}\right)^{6}\right]+\epsilon & \text { if } r \leq r_{0}, \\
0 & \text { if } r>r_{0},
\end{array}\right.
$$

where $r$ denotes the distance between two particles, $\epsilon$ and $\sigma$ are two positive parameters and $r_{0}=2^{1 / 6} \sigma$. Among these particles, two (numbered 1 and 2 in the following) are designated to form a dimer while the others are solvent particles. Instead of the above WCA potential, the interaction potential between the two particles of the dimer is a double-well potential

$$
V_{\mathrm{S}}(r)=h\left[1-\frac{\left(r-r_{0}-w\right)^{2}}{w^{2}}\right]^{2},
$$

\footnotetext{
${ }^{2}$ Of course, this threshold time depends on the system under study.
} 

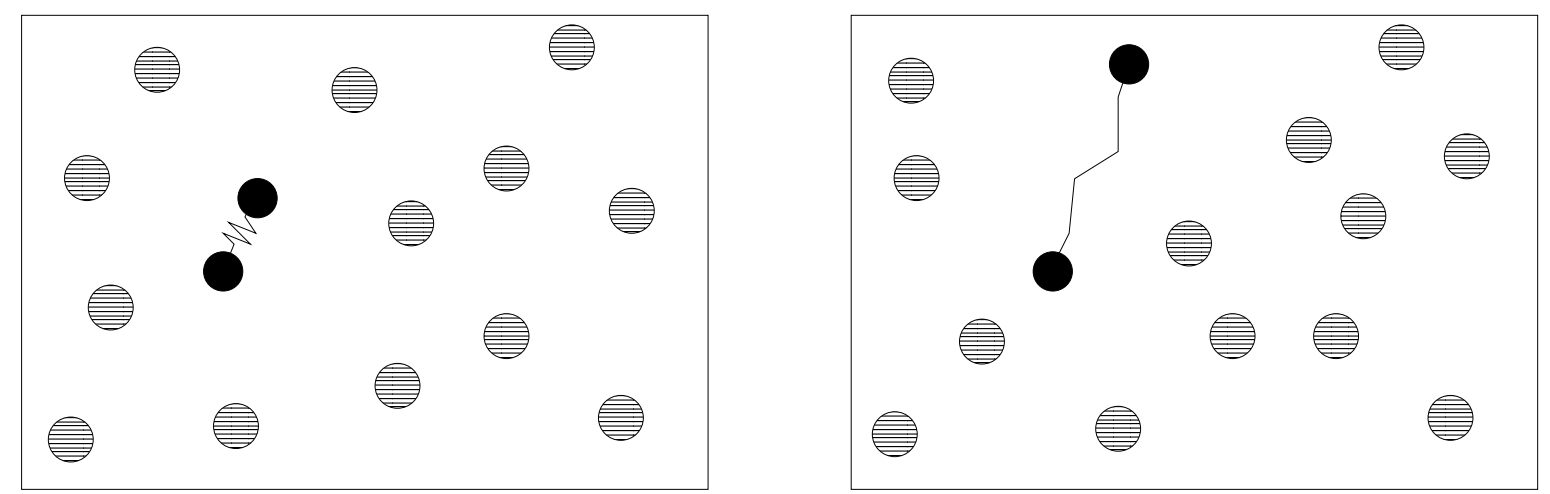

Figure 3: Schematic views of the system, when the dimer is in the compact state (Left), and in the stretched state (Right). The interaction of the particles forming the dimer is described by a double well potential. All the other interactions are of WCA form.

where $h$ and $w$ are two positive parameters. The potential $V_{\mathrm{S}}$ exhibits two energy minima, one corresponding to the compact state where the length of the dimer is $r=r_{0}$, and one corresponding to the stretched state where this length is $r=r_{0}+2 w$. The energy barrier separating both states is $h$. Figure 3 presents a schematic view of the system.

The reaction coordinate used is

$$
\xi(q)=\frac{\left|q_{1}-q_{2}\right|-r_{0}}{2 w}
$$

where $q_{1}$ and $q_{2}$ are the positions of the particles forming the dimer. The compact state (resp. the stretched state) corresponds to a value of the reaction coordinate $z=0$ (resp. $z=1$ ).

The parameters used for the simulations are: $\beta=1, \epsilon=1, \sigma=1, h=1, w=0.5$ and $N=$ 16. We still use a linear schedule: $z(t)=t / T$. The side length $l$ of the simulation box takes two values: $l=1.3$ (high density state) and $l=3$ (low density state). Figure 4 presents some plots of the free energy difference profiles computed using nonequilibrium dynamics, as well as thermodynamic integration reference profiles. The results show that nonequilibrium estimates are consistent with thermodynamic integration. Our experience on this particular example also shows that it is computationally as efficient to simulate several short nonequilibrium trajectories (provided the switching time is not too small, say, $T \sim 1$ in the units used here, so that the diffusion process can take place), or one single long trajectory where the switching is done slowly (as already observed in Section 4.1).

The free energy profiles highlight the relative stabilities of the two conformations of the dimer: at low densities (Figure 4, Left) the stretched conformation has a lower free energy and is thus expected to be more stable (this can indeed be verified by running long molecular dynamics trajectories and monitoring the time spent in each conformation). When the density increases, the compact conformation becomes more and more likely. At the density considered in Figure 4 (Right), the compact state already has a free energy slightly smaller than the stretched state. Notice also that the free energy barrier increases as the density increases, so that spontaneous transitions are less and less frequent. But since we know here a reaction coordinate, we can enforce the transition. This prevents us from running and monitoring long trajectories to get sufficient statistics to compare relative occurrences of both states. 

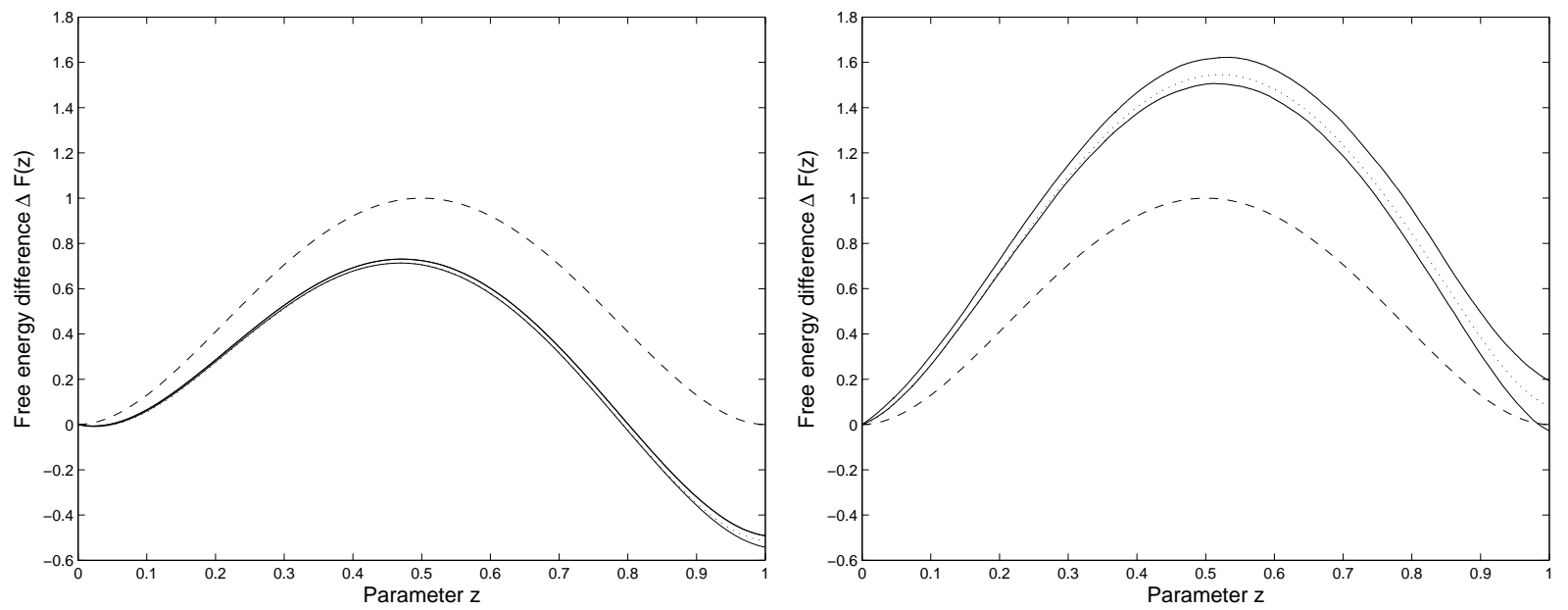

Figure 4: Comparison of free energy difference profiles using the reaction coordinate (39), at low densities $(l=3)$ on the left, and high densities $(l=1.3)$ on the right. The double well potential $V_{S}$ is represented in dashed line. The reference free energy difference profile computed with a very precise thermodynamic integration is represented in dotted line. We used $N_{\mathrm{TI}}=101$ thermodynamic integration points (uniformly distributed over $(0,1)$ ) and averaged the mean force over $M_{\mathrm{TI}}=10^{7}$ configurations for each fixed value of $z$. The upper and lower bounds of the $95 \%$ confidence interval (obtained over 50 independent realizations) for out-of-equilibrium computations are represented with solid lines. We used $M=1000$ nonequilibrium trajectories, a switching time $T=1$, and a timestep $\Delta t=0.00025$ (left) or $\Delta t=0.0005$ (right). 


\section{Acknowledgments}

The authors would like to thank the referees for their numerous comments to improve the paper. The authors would also like to thank the Centre International de Rencontres Mathématiques where this work has been partly performed. TL and GS acknowledge financial support from Action Concertée Incitative Nouvelles Interfaces des Mathématiques, "Simulation moléculaire", Ministère de la Recherche, France. Finally, part of this work was done while GS was attending the program "Bridging Time and Length Scales in Materials Science and Biophysics" at IPAM (UCLA).

\section{References}

[1] S.A. Allison and J.A. McCammon, Transport properties of rigid and flexible macromolecules by brownian dynamics simulation, Biopolymers 23(1) (1984) 167-187.

[2] H.C. Andersen, Rattle: a "velocity" version of the Shake algorithm for molecular dynamics calculations, J. Comput. Phys. 52 (1983) 24-34.

[3] J.F. Bonnans, J.C. Gilbert, C. Lemaréchal, and C.A. Sagastizábal. Numerical optimization. Springer, 2002.

[4] E.A. Carter, G. Ciccotti, J.T. Hynes, R. Kapral, Constrained reaction coordinate dynamics for the simulation of rare events, Chem. Phys. Lett. 156(5) (1989) 472-477.

[5] G. Ciccotti, R. Kapral, and E. Vanden-Eijnden, Blue Moon Sampling, Vectorial Reaction Coordinates, and Unbiased Constrained Dynamics, ChemPhysChem 6(9) (2005) 18091814.

[6] G. Ciccotti, T. Lelièvre, and E. Vanden-Eijnden, Projection of diffusions on submanifolds: Application to mean force computation, CERMICS 2006-309 preprint.

[7] E. Darve and A. Porohille, Calculating free energy using average forces, J. Chem. Phys., 115 (2001) 9169-9183.

[8] C. Dellago, P.G. Bolhuis, and D. Chandler, On the calculation of reaction rate constants in the transition path ensemble, J. Chem. Phys 110(14) (1999) 6617-6625.

[9] W. E, and E. Vanden-Eijnden, Metastability, conformation dynamics, and transition pathways in complex systems. Multiscale modelling and simulation, 35-68, Lect. Notes Comput. Sci. Eng., 39, Springer, Berlin, 2004.

[10] R. Glowinski and P. Le Tallec. Augmented Lagrangian and operator-splitting methods in nonlinear mechanics. Studies in Applied Mathematics. SIAM, 1989.

[11] D.A. Hendrix and C. Jarzynski, A "fast growth" method of computing free energy differences, J. Chem. Phys. 114(14) (2001) 5974-5981.

[12] J. Hénin and C. Chipot, Overcoming free energy barriers using unconstrained molecular dynamics simulations, J. Chem. Phys. 121 (2004) 2904-2914. 
[13] G. Hummer and A. Szabo, Free energy reconstruction from nonequilibrium singlemolecule pulling experiments, PNAS 98(7) (2001) 3658-3661.

[14] M. Iannuzzi, A. Laio and M. Parrinello, Efficient Exploration of Reactive Potential Energy Surfaces Using Car-Parrinello Molecular Dynamics, Phys. Rev. Lett. 90 (2003) 238302.

[15] C. Jarzynski, Equilibrium free energy differences from nonequilibrium measurements: a master equation approach, Phys. Rev. E 56(5) (1997) 5018-5035.

[16] C. Jarzynski, Nonequilibrium equality for free energy differences, Phys. Rev. Lett. 78(14) (1997) 2690-2693.

[17] J.G. Kirkwood, Statistical mechanics of fluid mixtures, J. Chem. Phys. 3 (1935) 300-313.

[18] B. Leimkuhler and S. Reich. Simulating Hamiltonian dynamics. Cambridge University Press, 2004.

[19] H. Oberhofer, C. Dellago, and P.L. Geissler, Biased sampling of non-equilibrium trajectories: Can fast switching simulations outperform conventional free energy calculation methods?, J. Chem. Phys. B 109 (2005) 6902-6915.

[20] H.C. Öttinger, Brownian dynamics of rigid polymer chains with hydrodynamic interactions, Phys. Rev. E 50(4) (1994) 2696-2701.

[21] W. K. den Otter and W. J. Briels, The calculation of free-energy differences by constrained molecular-dynamics simulations, J. Chem. Phys. 109(11) (1998) 4139-4146.

[22] S. Park, F. Khalili-Araghi, E. Tajkhorshid, and K. Schulten, Free energy calculation from steered molecular dynamics simulations using Jarzynski's equality, J. Chem. Phys. 119(6) (2003) 3559-3566.

[23] J.M. Rickman and R. LeSar, Free-energy calculations in materials research, Annu. Rev. Matter. Res. 32 (2002) 195-217.

[24] D. Rodriguez-Gomez, E. Darve and A. Pohorille, Assessing the efficiency of free energy calculation methods, J. Chem. Phys. 120 (2004) 3563-3578.

[25] M. Rousset and G. Stoltz, Equilibrium sampling from nonequilibrium dynamics, accepted for publication in J. Stat. Phys. (2006)

[26] J.P. Ryckaert, G. Ciccotti, and H.J.C. Berendsen, Numerical integration of the Cartesian equations of motion of a system with constraints: Molecular dynamics of $n$-alkanes, $J$. Comput. Phys. 23 (1977) 327-342.

[27] M. Sprik and G. Ciccotti, Free energy from constrained molecular dynamics, J. Chem. Phys. 109(18) (1998) 7737-7744.

[28] J.E. Straub, M. Borkovec, and B.J. Berne, Molecular dynamics study of an isomerizing diatomic in a Lennard-Jones fluid, J. Chem. Phys. 89(8) (1988) 4833-4847.

[29] A.M. Stuart, J. Voss, and P. Wiberg, Conditional path sampling of SDEs and the Langevin MCMC method, Commun. Math. Sci. 2(4) (2004) 685-697. 
[30] S. Sun, Equilibrium free energies from path sampling of nonequilibrium trajectories, $J$. Chem. Phys. 118(13) (2003) 5769-5775.

[31] G.M. Torrie and J.P. Valleau, Nonphysical sampling distributions in Monte-Carlo free energy estimation: Umbrella sampling, J. Comp. Phys. 23 (1977) 187-199.

[32] W.F. Van Gunsteren and H.J.C. Berendsen, Algorithms for brownian dynamics, Mol. Phys. 45(3) (1982) 637-647.

[33] A.F. Voter, A method for accelerating the molecular dynamics simulation of infrequent events, J. Chem. Phys. 106(11) (1997) 4665-4677.

[34] F.M. Ytreberg and D.M. Zuckerman, Single-ensemble nonequilibrium path sampling estimates of free energy differences, J. Chem. Phys. 120(3) (2004) 10876-10879.

[35] R. Zwanzig, High-temperature equation of state by a perturbation method: I. Nonpolar gases, J. Chem. Phys. 22 (1954) 1420-1426.

[36] D.M. Zuckerman and T.B. Woolf, Systematic finite sampling inaccuracy in free energy differences and other nonlinear quantities, J. Stat. Phys. 114(5-6) (2004) 1303-1323.

\section{A Appendix: The multi-dimensional case}

In this appendix, we generalize the previous results for nonequilibrium computation of free energy differences to the case of multi-dimensional reaction coordinates.

\section{A.1 Geometric setting and basic notation and formulae.}

We consider a $d$-dimensional system of smooth reaction coordinates $\xi=\left(\xi_{1}, \ldots, \xi_{d}\right): \mathbb{R}^{3 N} \rightarrow$ $\mathbb{R}^{d}$, non-singular on an open domain $\mathcal{M} \subset \mathbb{R}^{3 N}$

$$
\forall q \in \mathcal{M}, \quad \operatorname{range}\left(\nabla \xi_{1}(q), \ldots, \nabla \xi_{d}(q)\right)=d,
$$

and a smooth path of associated coordinates

$$
z=\left(z_{1}, \ldots, z_{d}\right):[0, T] \rightarrow \mathbb{R}^{d} .
$$

Accordingly, we define for all $t \in[0, T]$ a smooth submanifold of codimension $d$ contained in $\mathcal{M}$ :

$$
\Sigma_{z(t)}=\left\{q \in \mathbb{R}^{3 N}, \xi(q)=z(t)\right\} \subset \mathcal{M} .
$$

In the constraints space $\mathbb{R}^{d}$, coordinates are labeled by Greek letters and we use the summation convention on repeated indices. In the configuration space $\mathbb{R}^{3 N}$, coordinates are labeled by Latin letters and we also use the summation convention on repeated indices. We denote by $X \cdot Y=X_{i} Y_{i}$ the scalar product of two vector fields of $\mathbb{R}^{3 N}$, by $M: N=M_{i, j} N_{i, j}$ the contraction of two tensor fields of $\mathbb{R}^{3 N}$, and by $(X \otimes Y)_{i, j}=X_{i} Y_{j}$ the tensor product of two vector fields of $\mathbb{R}^{3 N}$.

The $d \times d$ matrix

$$
G_{\alpha, \gamma}=\nabla \xi_{\alpha} \cdot \nabla \xi_{\gamma}
$$


is the Gram matrix of the constraints. It is symmetric and strictly positive on $\mathcal{M}$. We denote by $G_{\alpha, \gamma}^{-1}$ the $(\alpha, \gamma)$ component of $G^{-1}$, the inverse matrix of $G$. At each point $q \in \mathcal{M}$, we define the orthogonal projection operator

$$
P^{\perp}=G_{\alpha, \gamma}^{-1} \nabla \xi_{\alpha} \otimes \nabla \xi_{\gamma}
$$

onto the normal space to $\Sigma_{\xi(q)}$ and the orthogonal projection operator

$$
P=\mathrm{Id}-P^{\perp}
$$

onto the tangent space to $\Sigma_{\xi(q)}$. The mean curvature vector field of the submanifold is defined by:

$$
H=-\nabla \cdot\left((\operatorname{det} G)^{1 / 2} G_{\alpha, \gamma}^{-1} \nabla \xi_{\gamma}\right)(\operatorname{det} G)^{-1 / 2} \nabla \xi_{\alpha}
$$

and satisfies:

$$
H_{i}=P_{j, k} \nabla_{j} P_{i, k} .
$$

We recall the divergence theorem on submanifolds: for any smooth function $\phi: \mathbb{R}^{3 N} \rightarrow$ $\mathbb{R}^{3 N}$ with compact support,

$$
\int_{\Sigma_{z}} \operatorname{div}_{\Sigma}(\phi) d \sigma_{\Sigma_{z}}=-\int_{\Sigma_{z}} H \cdot \phi d \sigma_{\Sigma_{z}}
$$

where $\operatorname{div}_{\Sigma}(\phi)=P_{i, j} \nabla_{i} \phi_{j}$ denotes the surface divergence, and $\sigma_{\Sigma_{z}}$ is the induced Lebesgue measure on the submanifold $\Sigma_{z}$ of $\mathbb{R}^{3 N}$.

We will also use the co-area formula: for any smooth function $\phi: \mathbb{R}^{3 N} \rightarrow \mathbb{R}$,

$$
\int_{\mathbb{R}^{3 N}} \phi(q)(\operatorname{det} G(q))^{1 / 2} d q=\int_{\mathbb{R}^{d}} \int_{\Sigma_{z}} \phi d \sigma_{\Sigma_{z}} d z .
$$

These definitions and formulae are provided with more details in [6].

\section{A.2 Free energy and constrained diffusions for multi-dimensional reaction coordinates}

As in the one-dimensional case, the Boltzmann-Gibbs distribution restricted on the submanifold $\Sigma_{z}$ is defined by:

$$
d \mu_{\Sigma_{z}}=Z_{z}^{-1} \exp (-\beta V) d \sigma_{\Sigma_{z}}
$$

with

$$
Z_{z}=\int_{\Sigma_{z}} \exp (-\beta V) d \sigma_{\Sigma_{z}}
$$

The associated free energy is:

$$
F(z)=-\beta^{-1} \ln \left(Z_{z}\right)
$$

Remark A.1 (On the definition of the free energy: the multi-dimensional case). As in the one-dimensional case (see Remark 1.1), if the particles initially evolve in a potential $V$, the classical definition of the free energy is as above, but with $V$ replaced by an effective potential $V+\beta^{-1} \ln \left((\operatorname{det} G)^{1 / 2}\right)$. The computation of the gradient of this potential in the dynamics then involves second-order derivatives of $\xi$, which can be approximated in practice by finite differences (see [6]). 
For any $1 \leq \alpha \leq d$, we now introduce the local mean force along $\nabla \xi_{\alpha}$ (which generalizes (11)):

$$
f_{\alpha}=G_{\alpha, \gamma}^{-1} \nabla \xi_{\gamma} \cdot\left(\nabla V+\beta^{-1} H\right) .
$$

As in the one-dimensional case (see Equation (100), we obtain the derivative of the mean force by averaging the local mean force:

Proposition A.2. The derivative of the free energy $F$ with respect to $z_{\alpha}$ is given by:

$$
\nabla_{\alpha} F(z)=\int_{\Sigma_{z}} f_{\alpha} d \mu_{\Sigma_{z}}
$$

Proposition A.2 is a corollary of

Lemma A.3. For any test function $\varphi$ with compact support in $\mathcal{M}$, we have:

$$
\nabla_{\alpha}\left(\int_{\Sigma_{z}} \varphi \exp (-\beta V) d \sigma_{\Sigma_{z}}\right)=\int_{\Sigma_{z}}\left(G_{\alpha, \gamma}^{-1} \nabla \xi_{\gamma} \cdot \nabla \varphi-\beta f_{\alpha} \varphi\right) \exp (-\beta V) d \sigma_{\Sigma_{z}} .
$$

Proof. It is enough to prove the formula in the case $V=0$, up to a modification of the test function $\varphi$. For any test function $g: \mathbb{R} \rightarrow \mathbb{R}$ with compact support, we have (using successively an integration by parts on $\mathbb{R}$, the co-area formula (42), an integration by parts on $\mathbb{R}^{3 N}$, and finally again (42)):

$$
\begin{aligned}
\int_{\mathbb{R}^{d}} g\left(z_{\alpha}\right) \nabla_{\alpha}\left(\int_{\Sigma_{z}} \varphi d \sigma_{\Sigma_{z}}\right) d z & =-\int_{\mathbb{R}^{d}} \int_{\Sigma_{z}} g^{\prime}\left(z_{\alpha}\right) \varphi d \sigma_{\Sigma_{z}} d z \\
& =-\int_{\mathbb{R}^{3 N}} g^{\prime} \circ \xi_{\alpha} \varphi(\operatorname{det} G)^{1 / 2} d q \\
& =-\int_{\mathbb{R}^{3 N}} G_{\alpha, \gamma}^{-1} \nabla \xi_{\gamma} \cdot \nabla\left(g \circ \xi_{\alpha}\right) \varphi(\operatorname{det} G)^{1 / 2} d q \\
& =\int_{\mathbb{R}^{3 N}} g \circ \xi_{\alpha} \nabla \cdot\left(G_{\alpha, \gamma}^{-1} \nabla \xi_{\gamma} \varphi(\operatorname{det} G)^{1 / 2}\right) d q \\
& =\int_{\mathbb{R}^{d}} g\left(z_{\alpha}\right) \int_{\Sigma_{z}} \nabla \cdot\left(G_{\alpha, \gamma}^{-1} \nabla \xi_{\gamma} \varphi(\operatorname{det} G)^{1 / 2}\right)(\operatorname{det} G)^{-1 / 2} d \sigma_{\Sigma_{z}} d z
\end{aligned}
$$

which gives the result using the expression (40) of the mean curvature vector $H$.

We now define the constrained diffusion (which generalizes (21)):

$$
\left\{\begin{array}{l}
Q_{0} \sim \mu_{\Sigma_{z(0)}} \\
d Q_{t}=-P\left(Q_{t}\right) \nabla V\left(Q_{t}\right) d t+\sqrt{2 \beta^{-1}} P\left(Q_{t}\right) \circ d B_{t}+\nabla \xi_{\alpha}\left(Q_{t}\right) d \Lambda_{\alpha, t}^{\mathrm{ext}} \\
d \Lambda_{\alpha, t}^{\mathrm{ext}}=G_{\alpha, \gamma}^{-1}\left(Q_{t}\right) z_{\gamma}^{\prime}(t) d t, \quad \forall 1 \leq \alpha \leq d
\end{array}\right.
$$

The stochastic process $Q_{t}$ can be characterized by the following property:

Proposition A.4. The process $Q_{t}$ solution to (44) is the only Itô process satisfying for some adapted Itô processes $\left(\Lambda_{1, t}, \ldots, \Lambda_{d, t}\right)_{t \in[0, T]}$ with values in $\mathbb{R}^{d}$ :

$$
\left\{\begin{array}{l}
Q_{0} \sim \mu_{\Sigma_{z(0)}} \\
d Q_{t}=-\nabla V\left(Q_{t}\right) d t+\sqrt{2 \beta^{-1}} d B_{t}+\nabla \xi_{\alpha}\left(Q_{t}\right) d \Lambda_{\alpha, t} \\
\xi\left(Q_{t}\right)=z(t)
\end{array}\right.
$$


Moreover, the process $\left(\Lambda_{\alpha, t}\right)_{t \in[0, T]}$ can be decomposed as

$$
\Lambda_{\alpha, t}=\Lambda_{\alpha, t}^{\mathrm{m}}+\Lambda_{\alpha, t}^{\mathrm{f}}+\Lambda_{\alpha, t}^{\mathrm{ext}},
$$

with the martingale part

$$
d \Lambda_{\alpha, t}^{\mathrm{m}}=-\sqrt{2 \beta^{-1}} G_{\alpha, \gamma}^{-1} \nabla \xi_{\gamma}\left(Q_{t}\right) \cdot d B_{t},
$$

the local force part (see [43) for the definition of $f_{\alpha}$ )

$$
d \Lambda_{\alpha, t}^{\mathrm{f}}=f_{\alpha}\left(Q_{t}\right) d t
$$

and the external forcing (or switching) term

$$
d \Lambda_{\alpha, t}^{\mathrm{ext}}=G_{\alpha, \gamma}^{-1}\left(Q_{t}\right) z_{\gamma}^{\prime}(t) d t .
$$

The proof consists in computing $d \xi\left(Q_{t}\right)$ by Itô's calculus and identifying the bounded variation and the martingale parts of the stochastic processes.

\section{A.3 The Feynman-Kac fluctuation equality}

Theorem 2.2 is generalized as:

Theorem A.5 (Feynman-Kac fluctuation equality). Let us define the nonequilibrium work exerted on the diffusion $Q_{t}$ solution to (44) by:

$$
\mathcal{W}(t)=\int_{0}^{t} f_{\alpha}\left(Q_{s}\right) z_{\alpha}^{\prime}(s) d s=\int_{0}^{t} z_{\alpha}^{\prime}(s) d \Lambda_{\alpha, s}^{\mathrm{f}} .
$$

Then, we have the following fluctuation equality: for any test function $\varphi$, and $\forall t \in[0, T]$,

$$
\frac{Z_{z(t)}}{Z_{z(0)}} \int_{\Sigma_{z(t)}} \varphi d \mu_{\Sigma_{z(t)}}=\mathbb{E}\left(\varphi\left(Q_{t}\right) \mathrm{e}^{-\beta \mathcal{W}(t)}\right) .
$$

In particular, we have the work fluctuation identity: $\forall t \in[0, T]$,

$$
\Delta F(z(t))=F(z(t))-F(z(0))=-\beta^{-1} \ln \left(\mathbb{E}\left(\mathrm{e}^{-\beta \mathcal{W}(t)}\right)\right) .
$$

Proof. For any $s \in[0, T]$ and $x \in \mathcal{M}$, let us introduce $\left(Q_{t}^{s, x}\right)_{t \in[s, T]}$, the stochastic process satisfying the SDE (44), starting from $x$ at time $s$ :

$$
\left\{\begin{array}{l}
Q_{s}^{s, x}=x \\
d Q_{t}^{s, x}=-P\left(Q_{t}^{s, x}\right) \nabla V\left(Q_{t}^{s, x}\right) d t+\sqrt{2 \beta^{-1}} P\left(Q_{t}^{s, x}\right) \circ d B_{t}+\nabla \xi_{\alpha}\left(Q_{t}^{s, x}\right) d \Lambda_{\alpha, t}^{\mathrm{ext}}, \\
d \Lambda_{\alpha, t}^{\text {ext }}=G_{\alpha, \gamma}^{-1}\left(Q_{t}^{s, x}\right) z_{\gamma}^{\prime}(t) d t, \quad \forall 1 \leq \alpha \leq d .
\end{array}\right.
$$

Notice that for any $s \in[0, T]$, there is an open neighborhood $\left(s^{-}, s^{+}\right) \times \mathcal{M}_{s}$ of $\left(s, \Sigma_{z(s)}\right)$ in $\mathbb{R} \times \mathcal{M}$ such that the diffusion $\left(Q_{t}^{s, x}\right)_{t \in[s, T]}$ remains in $\mathcal{M}$ almost surely. This holds since this process satisfies $d \xi\left(Q_{t}^{s, x}\right)=z^{\prime}(t) d t$ and therefore $\xi\left(Q_{t}^{s, x}\right)=\xi(x)+z(t)-z(s)$. This gives usual regularity assumptions sufficient to get a backward semi-group ( $t$ being from now on fixed in $(0, T)$ and $s$ varying in $[0, t])$ :

$$
u(s, x)=\mathbb{E}\left(\varphi\left(Q_{t}^{s, x}\right) \exp \left(-\beta \int_{s}^{t} f_{\alpha}\left(Q_{r}^{s, x}\right) z_{\alpha}^{\prime}(r) d r\right)\right),
$$


satisfying the following partial differential equation (PDE) on $\left(s^{-}, s^{+}\right) \times \mathcal{M}_{s}$ :

$$
\partial_{s} u=-L_{s}(u(s, .))+\beta z_{\alpha}^{\prime}(s) f_{\alpha} u,
$$

where $L_{s}$ is the generator of the diffusion $Q_{t}$ solution to (44):

$$
L_{s}=\beta^{-1} P: \nabla^{2}-P \nabla V \cdot \nabla+\beta^{-1} H \cdot \nabla+z_{\gamma}^{\prime}(s) G_{\alpha, \gamma}^{-1} \nabla \xi_{\alpha} \cdot \nabla .
$$

Now, using Lemma A.3, we have:

$$
\begin{aligned}
\frac{d}{d s} & \int_{\Sigma_{z(s)}} u(s, .) \exp (-\beta V) d \sigma_{\Sigma_{z(s)}} \\
& =\int_{\Sigma_{z(s)}}\left(-L_{s}(u(s, .))+z_{\alpha}^{\prime}(s) G_{\alpha, \gamma}^{-1} \nabla \xi_{\gamma} \cdot \nabla u(s, .)\right) \exp (-\beta V) d \sigma_{\Sigma_{z(s)}}, \\
& =-\int_{\Sigma_{z(s)}}\left(\beta^{-1} P: \nabla^{2} u(s, .)-P \nabla V \cdot \nabla u(s, .)+\beta^{-1} H \cdot \nabla u(s, .)\right) \exp (-\beta V) d \sigma_{\Sigma_{z(s)}} \\
& =-\beta^{-1} \int_{\Sigma_{z(s)}}\left(\operatorname{div}_{\Sigma}(\nabla u(s, .) \exp (-\beta V))+H \cdot \nabla u(s, .) \exp (-\beta V)\right) d \sigma_{\Sigma_{z(s)}}, \\
& =0
\end{aligned}
$$

by the divergence theorem (41). Therefore

$$
\int_{\Sigma_{z(t)}} u(t, .) \exp (-\beta V) d \sigma_{\Sigma_{z(t)}}=\int_{\Sigma_{z(0)}} u(0, .) \exp (-\beta V) d \sigma_{\Sigma_{z(0)}}
$$

which yields

$$
\int_{\Sigma_{z(t)}} \varphi \exp (-\beta V) d \sigma_{\Sigma_{z(t)}}=Z_{z(0)} \mathbb{E}\left(\varphi\left(Q_{t}\right) \exp \left(-\beta \int_{0}^{t} f_{\alpha}\left(Q_{r}\right) z_{\alpha}^{\prime}(r) d r\right)\right),
$$

where $Q_{t}$ satisfies (44). This proves (45), and (46) is obtained by taking $\varphi=1$.

\section{A.4 The numerical scheme}

The adaptation of the algorithm we propose for the one-dimensional case to the multidimensional case is straightforward. Indeed, the generalizations of schemes (29) and (30) to the multi-dimensional case are, respectively:

$$
\begin{gathered}
\left\{\begin{array}{l}
Q_{n+1}=Q_{n}-\nabla V\left(Q_{n}\right) \Delta t+\sqrt{2 \Delta t \beta^{-1}} U_{n}+\Delta \Lambda_{\alpha, n+1} \nabla \xi_{\alpha}\left(Q_{n+1}\right), \\
\text { where }\left(\Delta \Lambda_{\alpha, n+1}\right)_{1 \leq \alpha \leq d} \text { is such that } \xi\left(Q_{n+1}\right)=z\left(t_{n+1}\right),
\end{array}\right. \\
\left\{\begin{array}{l}
Q_{n+1}=Q_{n}-\nabla V\left(Q_{n}\right) \Delta t+\sqrt{2 \Delta t \beta^{-1}} U_{n}+\Delta \Lambda_{\alpha, n+1} \nabla \xi_{\alpha}\left(Q_{n}\right), \\
\text { where }\left(\Delta \Lambda_{\alpha, n+1}\right)_{1 \leq \alpha \leq d} \text { is such that } \xi\left(Q_{n+1}\right)=z\left(t_{n+1}\right) .
\end{array}\right.
\end{gathered}
$$

The force part $\Delta \Lambda_{\alpha, n}^{\mathrm{f}}$ of $\Delta \Lambda_{\alpha, n}$ is obtained by similar procedures as those described in Section 3.2. For example, the generalization of (31) is:

$$
\Delta \Lambda_{\alpha, n+1}^{\mathrm{f}}=\Delta \Lambda_{\alpha, n+1}-G_{\alpha, \gamma}^{-1}\left(Q_{n}\right)\left(z_{\gamma}\left(t_{n+1}\right)-z_{\gamma}\left(t_{n}\right)\right)+\sqrt{2 \Delta t \beta^{-1}} G_{\alpha, \gamma}^{-1} \nabla \xi_{\gamma}\left(Q_{n}\right) \cdot U_{n} .
$$

The generalization of (32) is also straightforward. 
Now, the estimator $\widehat{\Delta F}(z(T))$ of the free energy difference $\Delta F(z(T))$ is given by (34), with the following approximation of the work $\mathcal{W}(t)$ :

$$
\left\{\begin{array}{l}
\mathcal{W}_{0}=0, \\
\mathcal{W}_{n+1}=\mathcal{W}_{n}+\frac{z_{\alpha}\left(t_{n+1}\right)-z_{\alpha}\left(t_{n}\right)}{t_{n+1}-t_{n}} \Delta \Lambda_{\alpha, n+1}^{\mathrm{f}},
\end{array}\right.
$$

which generalizes (33). Notice that Remark 3.1 also holds for a multi-dimensional reaction coordinate. 